\title{
贵金属纳米粒子/硅纳米线复合纳米材料的制备及其催化性能研究
}

\author{
张晓丹 ${ }^{a, b}$ 田华 ${ }^{a}$ 贺军辉*,a 曹阳 ${ }^{a}$ \\ ( ${ }^{a}$ 中国科学院理化技术研究所 功能纳米材料实验室 北京 100190) \\ $\left(^{b}\right.$ 国家纳米科学中心 北京 100190)
}

\begin{abstract}
摘要 以十二烷基硫酸钠 $(\mathrm{SDS})$ 作为保护剂, 利用贵金属阳离子 $\left(\mathrm{M}^{n+}\right)$ 与 $\mathrm{HF}$ 处理后的硅纳米线 $(\mathrm{SiNWs})$ 之间的氧化还原 反应, 在 $\mathrm{SiNWs}$ 表面负载了贵金属纳米粒子. 通过调控 $\mathrm{SDS} / \mathrm{M}^{n+}$ 物质的量比、反应物浓度、反应温度等实验参数, 制 备出了金属纳米粒子粒径均一且负载密集的 AuNPs/SiNWs, PtNPs/SiNWs 复合材料. 将 AuNPs/SiNWs 复合材料应用在 亚甲基蓝的还原反应，实验结果显示, $60 \mathrm{~min}$ 内 $\mathrm{AuNPs} / \mathrm{SiNWs}$ 可以将 55\%的亚甲基蓝还原，表明 AuNPs/SiNWs 具有良 好的催化活性. 这种复合材料易于从反应溶液中分离出来, 可以实现纳米催化剂的循环使用.
\end{abstract}

关键词 硅纳米线; 贵金属纳米粒子; 亚甲基蓝; 催化剂

\section{Synthesis and Catalytic Activity of Silicon Nanowires Decorated with Noble Metal Nanoparticles}

\author{
Zhang, Xiaodan ${ }^{a, b} \quad{\text { Tian, } \text { Hua }^{a} \quad \mathrm{He} \text {, Junhui* }}^{*, a}$ Cao, Yang ${ }^{a}$ \\ ( ${ }^{a}$ Functional Nanomaterials Laboratory, Technical Institute of Physics and Chemistry, Chinese Academy of Sciences, \\ Beijing 100190) \\ ( ${ }^{b}$ National Center for Nanoscience and Technology, Beijing 100190)
}

\begin{abstract}
In this paper, a new nanomaterial catalyst for methylene blue reduction was prepared. The design rationale is explained as follows: (1) silicon nanowires (SiNWs) show a number of remarkable advantages, such as easy surface modification with various noble metal nanoparticles (NMNPs), large surface-to-volume ratio, and stability to atmosphere environment. However, metal particles usually have large size and low density because of their aggregation; (2) Sodium dodecyl sulfate (SDS) is used as stabilizer, therefore metal nanoparticle size can be effectively controlled; furthermore, due to the weak-interaction between SiNWs and NMNPs, the catalytic activity may be mantained. Therefore, it is anticipated that the SiNWs can be modified by NMNPs with high density so as to become high efficient catalysts. In details, SiNWs were grown on $\mathrm{Si}$ wafers by thermal evaporation of $\mathrm{SiO}$ powder at $1350{ }^{\circ} \mathrm{C}$ for $6 \mathrm{~h}$, under a 300 Torr flowing gas mixture of $5 \% \mathrm{H}_{2}-\mathrm{Ar}$. The as-prepared SiNWs were etched 3 min with aqueous HF to remove their surface oxide layer. With SDS as protector, noble metal nanoparticles (NMNPs) were loaded on silicon nanowires (SiNWs) via the redox reaction between $\mathrm{M}^{n+}$ and HF-treated SiNWs for 5 minutes. In order to gain AuNPs/SiNWs composites with high coverage density and uniform size of AuNPs, experiment parameters were adjusted, such as the SDS/Au molar ratio $(200: 1,1000: 1,1200: 1)$, the $\mathrm{HAuCl}_{4}$ concentration $\left(1.0 \times 10^{-5}, 2.5 \times 10^{-5}, 4.0 \times 10^{-5} \mathrm{~mol} / \mathrm{L}\right)$, and reaction temperature $\left(15,30{ }^{\circ} \mathrm{C}\right)$. Meanwhile, Different NMNPs/SiNWs composites, such as PtNPs/SiNWs and AgNPs/SiNWs were also prepared. Finally, AuNPs/SiNWs were used in the reduction of methylene blue in the presence of sodium borohydride. In a representative reduction experiment, 0.1 $\mathrm{mL}$ of methylene blue solution $\left(2.5 \times 10^{-3} \mathrm{~mol} / \mathrm{L}\right)$ was mixed with $2.8 \mathrm{~mL}$ of $\mathrm{Na}_{2} \mathrm{CO}_{3} / \mathrm{NaHCO}_{3}$ buffer solution $(\mathrm{pH}=9.5)$, and purged with $\mathrm{N}_{2}$ gas for $5 \sim 6 \mathrm{~min}$ to remove all dissolved oxygen. The solution showed a $\lambda_{\max }$ at $664 \mathrm{~nm}$. Then $5 \mathrm{mg}$ the as-prepared catalysts and $0.15 \mathrm{~mL} 0.01 \mathrm{~mol} / \mathrm{L}$ of freshly prepared sodium borohydride were added. The progress of the reaction was monitored by a spectrophotometer. As a result, 55\% methylene blue was reduced in 60 min, which showed AuNPs/SiNWs had excellent catalytic activity. And it was found that $62 \%$ methylene blue was reduced in 100 min, and the reduction was going on even after 160 min reaction. These composites could be separated from reaction solution, thus ensuring recycling of nanocatalysts.
\end{abstract}

Keywords silicon nanowires; noble metal nanoparticles; methylene blue; catalyst

\section{1 引言}

\section{人们对贵金属纳米粒子(NMNPs)作为无机或有机}

的反应催化剂已进行了广泛研究. 一方面, 纳米微粒的 催化效率会随着尺寸的增加而降低，但是另一方面它们 高的表面能使得纳米微粒表面能倾向于变小而出现团

*E-mail: jhhe@mail.ipc.ac.cn

Received December 19, 2012; published January 31, 2013.

Supporting information for this article is available free of charge via the Internet at http://sioc-journal.cn.

Project supported by the National Basic Research Program of China (Grant No. 2006CB933000).

项目受科技部重大科学研究计划(No. 2006CB933000)资助. 
聚, 所以如何对其尺寸进行有效调控, 防止纳米粒子的 聚集显得尤为重要. 为了解决这个问题, 纳米粒子的催 化剂通常会负载在固体的载体上, 例如碳 ${ }^{[1,2]}$ 、钛 ${ }^{[3]}$ 、 银 ${ }^{[4]}$ 、氧化铝 ${ }^{[5]}$ 、二氧化硅球 ${ }^{[6]}$ 、聚苯乙烯球 ${ }^{[7]}$ 、沸石 ${ }^{[8]}$ 以及蒙脱石 ${ }^{[9]}$ 等.

硅纳米线由于表面易于修饰各种金属粒子，以及大 的比表面积、良好的稳定性, 引起了人们的极大兴 趣 ${ }^{[10 \sim 14]}$. 经 HF 处理, SiNWs 形成富含还原性的 $\mathrm{Si}-\mathrm{H}$ 键的表面, 进而将金属离子 $\left(\mathrm{M}^{n+}\right)$ 还原为金属纳米粒子, 从而形成稳定的金属纳米粒子与 SiNWs 的复合体, 该 复合材料不仅能够有效防止金属纳米粒子的聚集，而且 反应后催化剂易于从反应溶液中分离出来, 在催化反应 领域具有很好的应用前景. 但是修饰过程中未加保护剂 制得的金属纳米粒子仍存在粒径大、尺寸分布不均一、 负载密度小等缺点 ${ }^{[13,15,16]}$.

Zhang 等 ${ }^{[17]}$ 采用正己硫醇作为保护剂制备金纳米粒 子/SiNWs 复合材料, 有效控制了金纳米粒子(AuNPs)粒 径, 实现了粒子尺寸均一且负载的密集, 但是保护剂正 己硫醇是通过共价键与 AuNPs 作用, 这会使金属表面 活性位减小, 使 AuNPs 的表面催化活性降低, 从而限制 了该复合材料在催化方面的应用 ${ }^{[18]}$. 十二烷基硫酸钠 (SDS)作为一种保护剂, 是以松散的结构包围在纳米粒 子催化剂表面, 不但与正己硫醇一样可以有效地控制 AuNPs 的粒径, 且它与 AuNPs 之间不是通过共价键相 连, 对 AuNPs 自身的表面性能影响很小, 使 AuNPs 很好 的保持了自身的催化活性 ${ }^{[19 ~ 25]}$.

本文首次在 SiNWs 的负载过程中引入保护剂 SDS, 合成了 NMNPs/SiNWs 复合材料. 研究了负载过程中反 应物浓度、SDS 含量、反应温度等实验条件对产物形貌 的影响, 并将其用于亚甲基蓝的催化反应中, 考察该复 合材料的催化性能.

\section{2 结果与讨论}

\subsection{HF 处理前后 SiNWs 的结构表征}

SiNWs 的制备采用的是热蒸发法, 图 1 是制得的 SiNWs 与 HF 处理后 SiNWs 的 TEM 图像: SiNWs 的直 径约 $20 \mathrm{~nm}$, 长达数微米; HF 处理后的 SiNWs 由于外面 的氧化层被去除, 直径为 $12 \mathrm{~nm}$ 左右. 图 $1 \mathrm{~b}$ 中的插图是 $\mathrm{HF}$ 处理后 SiNWs 的选区电子衍射图(SAED), 其中 $\mathrm{Si}$ 有较明显的衍射点, 分别是 $\operatorname{Si}(220), \operatorname{Si}(111)$ 和 $\operatorname{Si}(311)$.

\section{2 保护剂 SDS 对负载的影响}

图 2 为不同 $\mathrm{SDS} / \mathrm{Au}$ 物质的量比制备的 AuNPs/SiNWs 材料的 TEM 图像. 从 AuNPs 的尺寸看, $\mathrm{SDS} / \mathrm{Au}$ 物质的量比分别为 $200: 1,1000: 1$ 以及 $1200: 1$ 的 3 个样品, AuNPs 的粒径先减小后增大; 从覆 盖的效果来看, 负载在 SiNWs 上的 AuNPs 逐渐减少. 这 是因为随着 SDS 含量的增加有更多的 SDS 分子覆盖到
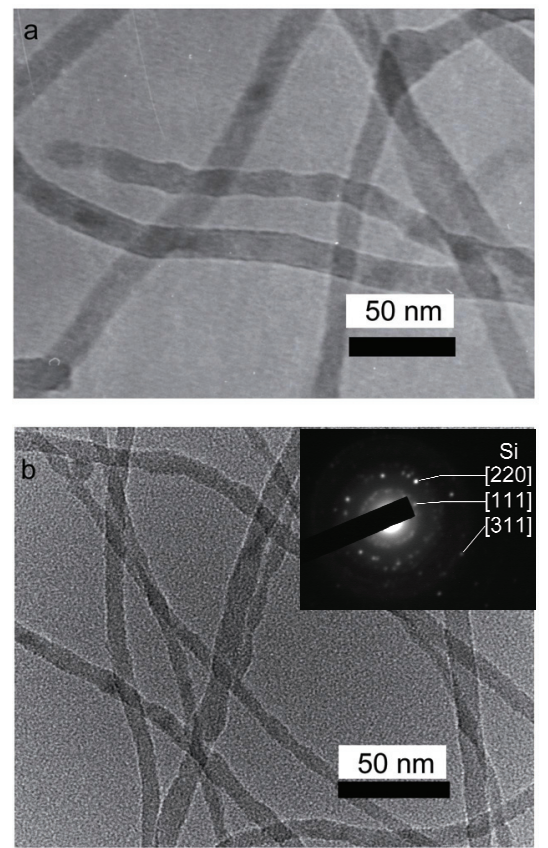

图 1 制得的 SiNWs (a) 和 HF 处理后 SiNWs (b)的 TEM 图像. (b)中插 图是 HF 处理后 SiNWs 的选区电子衍射(SAED) 图像

Figure 1 TEM images of as-prepared (a) and HF-treated SiNWs. The illustration in (b) is SAED pattern of HF-treated SiNWs

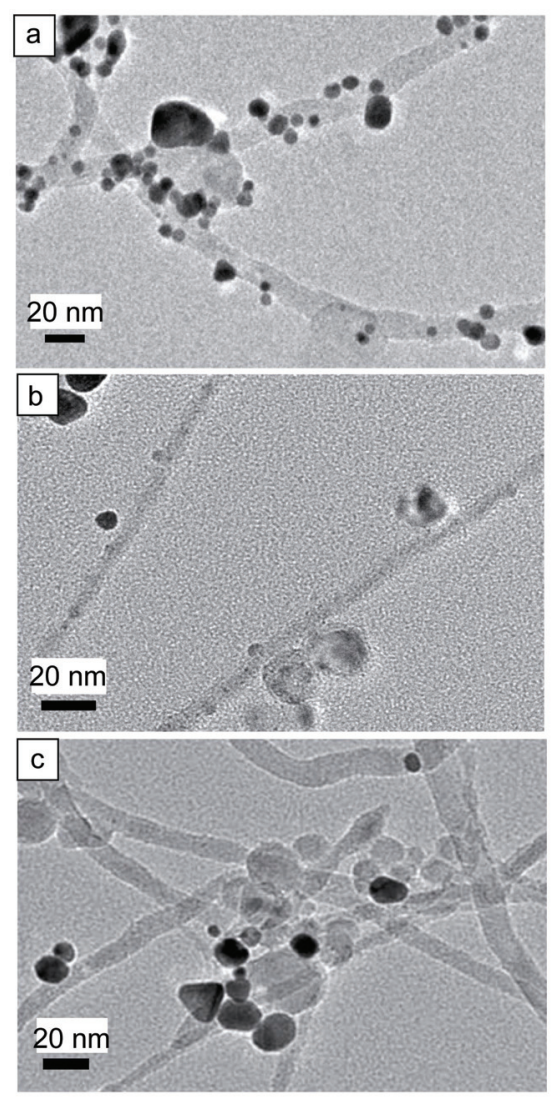

图 2 不同 $\mathrm{SDS} / \mathrm{Au}$ 物质的量比合成的 AuNPs/SiNWs 复合材料的 TEM 图像. 反应条件为: $5.4 \mathrm{mg} \mathrm{SiNWs,} 25 \mathrm{~mL} 10^{-4} \mathrm{~mol}^{\prime} / \mathrm{L} \mathrm{HACl}_{4}, \mathrm{SDS} / \mathrm{Au}$ 物质的量比分别为 $200: 1$ (a), 1000:1 (b), $1200: 1$ (c)

Figure 2 TEM images of AuNPs/SiNWs composite materials prepared with different SDS/Au ratio: $200: 1$ (a), $1000: 1$ (b) and $1200: 1$ (c) 
了金的表面, 限制了金粒子的进一步生长 ${ }^{[23,24]}$, 粒径较 小的粒子更易于稳定在 SiNWs 的表面，这也证明了 SDS 可以有效地控制纳米粒子的聚集; 但随着 SDS 含 量的进一步增加, 表面活性剂 SDS 由于浓度过高而发 生聚集，不能很好的稳定和控制金纳米粒子的粒径，使 金纳米粒子发生了团聚而长成大的颗粒, 并且因尺寸较 大易于从 SiNWs 上脱落下来 ${ }^{[26]}$. 由此可见, SDS 含量对 金属纳米粒子的负载具有很大影响, 适量的 SDS 可以 起到控制 AuNPs 的进一步生长, 防止团聚的作用; 但是 过量的 SDS 不仅不能起到控制粒径的作用, 还会对原 有的催化剂造成污染. 本文选择 SDS/Au 物质的量比为 $200: 1$ 进行以下的研究.

\section{3 反应温度的影响}

从图 3 的 $\mathrm{TEM}$ 图可以看出, 在 $\mathrm{HAuCl}_{4}$ 浓度为 $1.5 \times 10^{-5} \mathrm{~mol} / \mathrm{L}$ 的条件下, 当反应温度为 $15{ }^{\circ} \mathrm{C}$ 时, SiNWs 上只能修饰上很少的 AuNPs, 这说明较低的温度 不利于金属纳米粒子的负载; 当温度升高到 $30{ }^{\circ} \mathrm{C}$ 时, SiNWs 上的 AuNPs 获得了较密集的负载, 修饰效果获 得了很大的提高. AuNPs 在 SiNWs 上的负载过程实际上 是一个氧化还原反应的过程, 温度能够影响氧化反应的 进行, 必然影响负载效果 ${ }^{[27,28]}$. 随着温度的上升, 反应 加速进行, 这在负载效果上表现为: 很短的时间里金属 离子在 SiNWs 表面快速的大量成核, 进而快速长成大 量的粒径均一的 AuNPs, 形成负载良好的 AuNPs/ SiNWs 复合物. 同时, 在实验过程中发现过高的温度也 会导致形成粒径较大的粒子, 容易从 SiNWs 表面脱落 下来. 以下实验数据中选择 $30{ }^{\circ} \mathrm{C}$ 作为反应的温度.

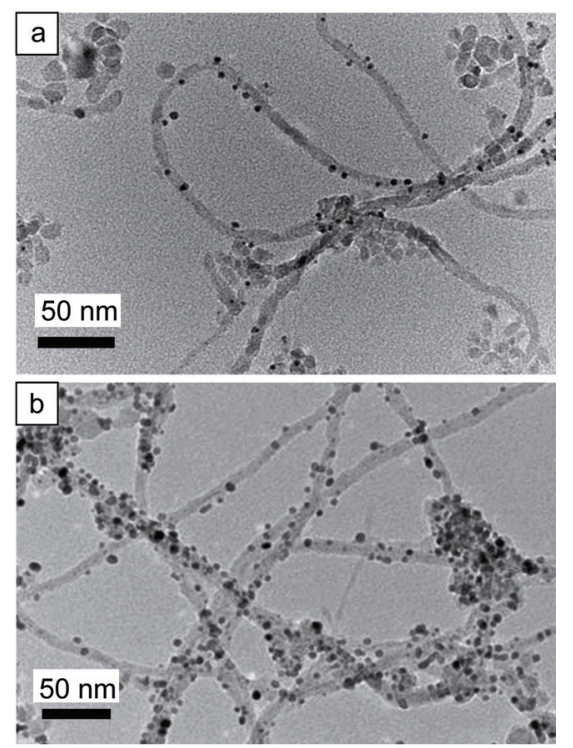

图 3 温度分别为 $15{ }^{\circ} \mathrm{C}$ (a) 和 $30^{\circ} \mathrm{C}($ b)下制备的 AuNPs/SiNWs 复合物 的 TEM 图像

Figure 3 TEM images of AuNPs/SiNWs prepared at the reaction temperature of $15{ }^{\circ} \mathrm{C}$ (a) and $30{ }^{\circ} \mathrm{C}$ (b)

Prepared condition: $5 \mathrm{mg} \mathrm{SiNWs,} 15 \mathrm{~mL} 1.5 \times 10^{-5} \mathrm{~mol} / \mathrm{L} \mathrm{HAuCl}_{4}$, SDS/Au ratio $=200: 1$

\section{$2.4 \mathrm{HAuCl}_{4}$ 浓度的影响}

我们考察了不同 $\mathrm{HAuCl}_{4}$ 浓度下 $\mathrm{AuNPs} / \mathrm{SiNWs}$ 复合 材料的制备情况, 如图 4 所示. 图 4c 中的插图是 $2.5 \times$ $10^{-5} \mathrm{~mol} / \mathrm{L} \mathrm{HAuCl}_{4}$ 条件下制备的 AuNPs/SiNWs 复合材 料的选区电子衍射图(SAED). 其中 $\mathrm{Si}$ 和 $\mathrm{Au}$ 都有明显的 衍射点, 分别是 $\mathrm{Si}(110), \mathrm{Si}(220)$ 和 $\mathrm{Si}(311), \mathrm{Au}(220)$ 和 $\mathrm{Au}(111)$, 晶面对应的衍射环也很明显, 这证实了 AuNPs 在 SiNWs 表面上的负载. 当 $\mathrm{HAuCl}_{4}$ 浓度分别为 $1.0 \times 10^{-5}, 2.5 \times 10^{-5}, 4.0 \times 10^{-5} \mathrm{~mol} / \mathrm{L}$ 时, 得到 AuNPs 的粒径分别为 $3.2 \pm 0.9,4.8 \pm 1.0$ 以及 $5.6 \pm 1.0 \mathrm{~nm}$.

由图 4 中的 TEM 图可知, 随着 $\mathrm{HAuCl}_{4}$ 浓度从 $1.0 \times 10^{-5} \mathrm{~mol} / \mathrm{L}$ 增加到 $2.5 \times 10^{-5} \mathrm{~mol} / \mathrm{L}, \mathrm{AuNPs}$ 的粒径 变大、覆盖度增加. 当浓度从 $2.5 \times 10^{-5} \mathrm{~mol} / \mathrm{L}$ 进一步增 大到 $4.0 \times 10^{-5} \mathrm{~mol} / \mathrm{L}, \mathrm{AuNPs}$ 的粒径继续增大, 但它在 SiNWs 上的覆盖密度明显减小. 这表明控制 $\mathrm{HAuCl}_{4}$ 浓 度也可以直接影响到负载效果: 当 $\mathrm{HAuCl}_{4}$ 为 $1.0 \times 10^{-5}$ $\mathrm{mol} / \mathrm{L}$ 时, 由于溶液中 $\mathrm{HAuCl}_{4}$ 的浓度较低, 导致负载到 SiNWs 上的 AuNPs 的数量较少; $\mathrm{HAuCl}_{4}$ 浓度为 $2.5 \times$ $10^{-5} \mathrm{~mol} / \mathrm{L}$ 时, 扩散到 $\mathrm{SiNWs}_{\mathrm{n}}$ 責的 $\mathrm{HAuCl}_{4}$ 明显增多, 使得 AuNPs 覆盖密度增大; 但当 $\mathrm{HAuCl}_{4}$ 的浓度进一步 增大时, 刚被还原了的 AuNPs 聚集成为尺寸较大的 AuNPs, 很容易从 SiNWs 的表面脱落下来, 导致负载密 度的进一步降低 ${ }^{[29 \sim 32]}$.

从上面的结果可知, $\mathrm{HAuCl}_{4}$ 浓度在 $\mathrm{AuNPs} / \mathrm{SiNWs}$ 复合材料的制备过程中也是一个重要的参数, 所以调整 $\mathrm{HAuCl}_{4}$ 浓度在制备负载密集和粒径均一的复合材料方 面具有重要的意义.

\section{5 不同金属在 SiNWs 上的负载}

为了考察不同金属纳米粒子在 SiNWs 表面上的负 载情况, 本文不仅制备了 AuNPs/SiNWs, 也制备了 PtNPs/SiNWs 和 $\mathrm{AgNPs} / \mathrm{SiNWs}$ 纳米材料. 在其它制备 条件一致的情况下, 以 $\mathrm{HAuCl}_{4}$ 和 $\mathrm{H}_{2} \mathrm{PtCl}_{4}$ 为原料, 制备 了 AuNPs/SiNWs 和 PtNPs/SiNWs 复合物, 如图 5 所示. 在 PtNPs 的负载中, 几乎每根 SiNWs 上都有非常密集的 负载; 而 AuNPs 的负载中，有少数 SiNWs 上没有金属粒 子, 这说明 Pt 比 $\mathrm{Au}$ 更容易与 SiNWs 相复合.

除了 PtNPs, 实验中也进行了 $\mathrm{Ag}$ 纳米粒子在 SiNWs 上的负载, 但是可能是因为 AgNPs 更加容易团 聚的缘故, AgNPs 负载量小, 粒径大, 分布不均一. 如图 $\mathrm{S} 1$, 可见 $\mathrm{Ag}$ 纳米粒子的负载效果远不如 $\mathrm{Au}$ 和 $\mathrm{Pt}$ 的.

\subsection{SiNWs/AuNPs 催化降解亚甲基蓝}

在亚甲基蓝催化反应中, 我们选用了图 4c 中的样 品为催化剂. 从图 4 中的 TEM 可以看出 SiNWs 的质量 较好, 负载均匀. 从它的粒径分布柱形图(图 4d)可以看 出, AuNPs 的直径约为 $4.8 \mathrm{~nm}$, 根据相关报道这样的尺 寸的 AuNPs 有比较好的催化活性 ${ }^{[16,19]}$. 亚甲基蓝的催化 还原反应结果如图 6 所示. 

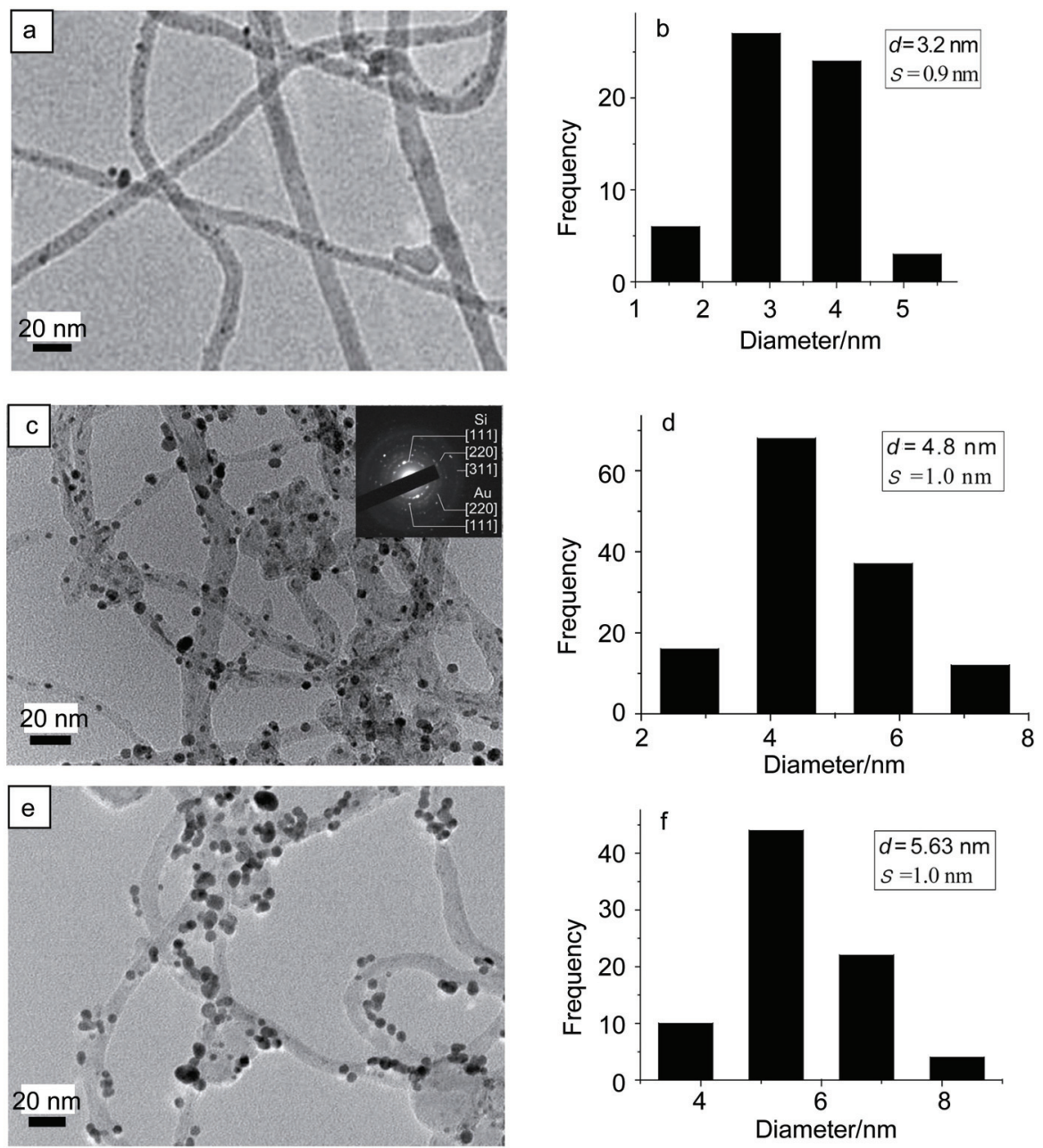

图 4 不同 $\mathrm{HAuCl}_{4}$ 浓度下合成的 $\mathrm{AuNPs} / \mathrm{SiNWs}$ 复合材料的 TEM 图像

Figure 4 TEM images of AuNPs/SiNWs

Prepared condition: $5.2 \mathrm{mg} \mathrm{SiNWs,} 15 \mathrm{~mL} 1.0 \times 10^{-5} \mathrm{~mol} / \mathrm{L}$ (a), $2.5 \times 10^{-5} \mathrm{~mol} / \mathrm{L}$ (c), $4.0 \times 10^{-5} \mathrm{~mol} / \mathrm{L}$ (e) $\mathrm{HAuCl}_{4}$; SDS/Au ratio of $200: 1$. (b), (d), (f) are size-distribution-histograms of AuNPs of (a), (c), (e), respectively. The illustration in (c) is SAED pattern of AuNPs/SiNWs.
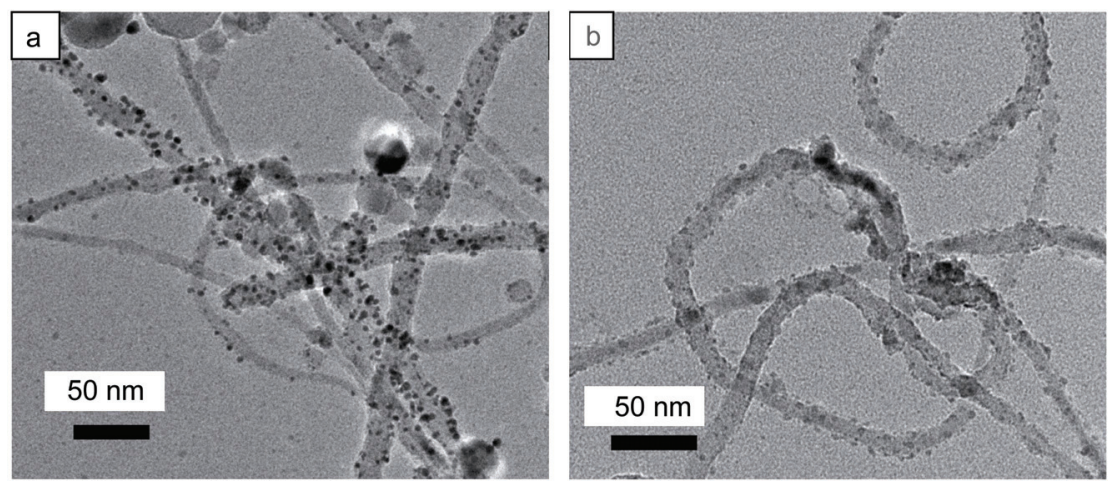

图 $5 \mathrm{AuNPs} / \mathrm{SiNWs}(\mathrm{a})$ 和 PtNPs/SiNWs (b)复合物的 TEM 图像

Figure 5 TEM images of AuNPs/SiNWs (a) and PtNPs/SiNWs (b)

Prepared condition: $5 \mathrm{mg}$ SiNWs, $15 \mathrm{~mL} 2.5 \times 10^{-5} \mathrm{~mol}^{2} / \mathrm{HAuCl}_{4}$ (a) and $2.5 \times 10^{-5} \mathrm{~mol} / \mathrm{L} \mathrm{H}_{2} \mathrm{PtCl}_{4}$ (b), SDS/Au or SDS/Pt ratio of $200: 1$

由图 6 所示, 610 和 $665 \mathrm{~nm}$ 两处为亚甲基蓝的特征 吸收峰, 在加入催化剂和还原剂 $\mathrm{NaBH}_{4}$ 后缓慢降低, 这 是因为亚甲基蓝发生了催化反应，由一种氧化态变成了 一种还原态. 随着反应的进行, 亚甲基蓝的特征吸收峰 强度在不断降低. 当反应 $60 \mathrm{~min}$ 以后, 吸收值由开始的
3.06 降到 1.38, 说明亚甲基蓝还原了 55\%. 进一步延长 反应时间(图 S2), 在反应进行到 $100 \mathrm{~min}$ 时, 亚甲基蓝 被还原了 $62 \%$, 这说明亚甲基蓝的催化反应还在进行.

根据朗伯比尔定律: $A=\lg (1 / T)=K b c, K$ 为比例常 数, $A$ 为吸光度, $T$ 为透射比, $c$ 为吸光物质的浓度, $b$ 为吸 


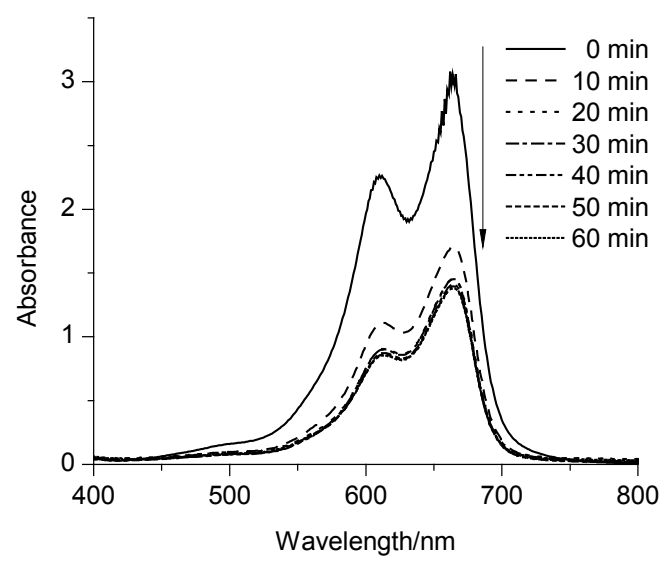

图 $6 \mathrm{AuNPs} / \mathrm{SiNWs}$ 催化还原亚甲基蓝的反应过程的 UV-Vis 吸收光 谱

Figure 6 UV-Vis spectra of methylene blue reduction with AuNPs/SiNWs as catalyst

Reaction condition: $0.1 \mathrm{~mL} 2.5 \times 10^{-3} \mathrm{~mol} / \mathrm{L}$ methylene blue, $5 \mathrm{mg}$ AuNPs/SiNWs, $0.15 \mathrm{~mL} 0.01 \mathrm{~mol} / \mathrm{L} \mathrm{NaBH}_{4}$.

收层厚度. 依据这个定律进一步将图 6 中的数据处理成 亚甲基蓝转化率与时间的关系曲线(图 7). 从这个曲线 可以更准确看出, 在亚甲基蓝的催化反应中前 $20 \mathrm{~min}$ 还 原了 $44 \%$, 随着时间的进行催化反应的速度变慢; 到反 应时间达到 $60 \mathrm{~min}$ 时，亚甲基蓝共还原了 55\%。

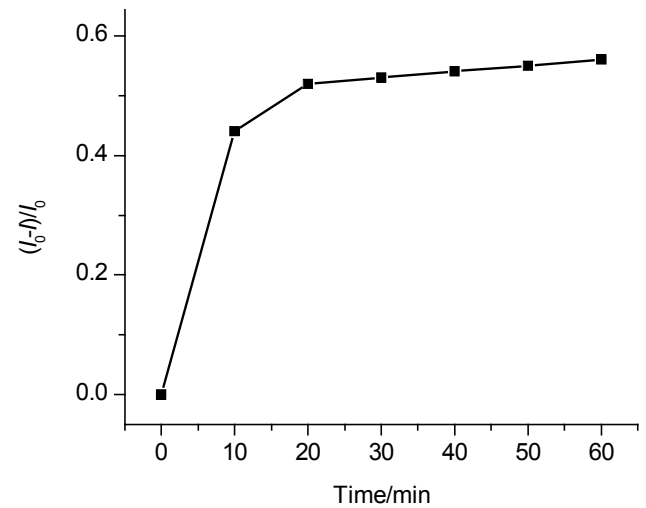

图 7 亚甲基蓝转化率对时间作图

Figure 7 Conversion of methylene blue with different reaction time

Reaction condition: $0.1 \mathrm{~mL} 2.5 \times 10^{-3} \mathrm{~mol} / \mathrm{L}$ methylene blue, $5 \mathrm{mg}$ AuNPs/SiNWs, $0.15 \mathrm{~mL} 0.01 \mathrm{~mol} / \mathrm{L} \mathrm{NaBH}_{4}$

与以往报道的 AuNPs 纳米催化剂相比, 本文合成 的负载型 AuNPs 是负载在微米级长度的硅纳米线上, 不易脱落, 有利于 $\mathrm{Au}$ 催化剂的分离和重复利用 ${ }^{[16,33]}$. 在实验中, 将已进行过反应的催化剂经过过滤洗涤, 重 复实验 3 次, $60 \mathrm{~min}$ 内亚甲基蓝分别降解了 $53.4 \%$, $51.2 \%$ 和 $49.0 \%$, 显示了较好的催化活性.

\section{3 结论}

本文使用 SDS 作为保护剂, SiNWs 作为载体合成了 $\mathrm{NMNPs} / \mathrm{SiNWs}$ 复合材料. 通过调整负载过程中 $\mathrm{HAuCl}_{4}$ 浓度、SDS 含量、反应温度等实验参数, 获得了纳米粒
子粒径小、尺寸分布均一、负载密集的 NMNPs/SiNWs 复合材料. 将 AuNPs 负载的 AuNPs/ SiNWs 复合材料作 为催化剂应用在亚甲基蓝的降解反应中, 经过 $60 \mathrm{~min}$, 55\%的亚甲基蓝获得了还原，表现出了很好的催化效 果. 这种复合材料不仅具有易于分离、可重复利用的优 点而且具有良好的催化活性, 因此是一种很好的催化材 料, 这在催化反应中具有重要的现实意义.

\section{4 实验部分}

\subsection{NMNPs/SiNWs 复合材料的制备}

热蒸发法制备的 SiNWs 具有产量大, 纯度高, 尺寸 均匀, 无催化剂污染的优点 ${ }^{[32}$ 35], 本文采用了该法制备 SiNWs. 制备过程如下：首先将装有原料 $\mathrm{SiO}$ 粉末的 $\mathrm{Al}_{2} \mathrm{O}_{3}$ 的瓷舟放在真空管式炉的中央, 同时在炉子的尾 端处放一个 $\mathrm{Al}_{2} \mathrm{O}_{3}$ 接受祄底, 以收集生长出来的 SiNWs. 密闭系统后, 抽真空到 $10^{-1} \mathrm{~Pa}$ 左右, 以 $50 \mathrm{~cm}^{3} / \mathrm{min}$ 的速 度通入 $\mathrm{H}_{2}$ 和 $\operatorname{Ar}\left(5 \% \mathrm{H}_{2}, 95 \% \mathrm{Ar}\right)$ 的混合气以保持 SiNWs 生长中的情性气氛, 气压达到 300 Torr 左右以 $10{ }^{\circ} \mathrm{C} / \mathrm{min}$ 的速度开始升温. 最后在 $1350{ }^{\circ} \mathrm{C}$ 密封的状态下保持 $6 \mathrm{~h}$, 自然降温.

金属纳米粒子/SiNWs 的制备: 配制一定 $\mathrm{SDS} /$ 金属 离子物质的量比 $\left(\mathrm{SDS} / \mathrm{M}^{n+}\right)$ 的金属离子 $\left(\mathrm{HAuCl}_{4}, \mathrm{AgNO}_{3}\right.$, $\left.\mathrm{H}_{2} \mathrm{PtCl}_{4}\right)$ 与 $\mathrm{SDS}$ 的水溶液, 金属离子的浓度为 $10^{-5} \sim$ $10^{-2} \mathrm{~mol} / \mathrm{L}$; 最后将一定量的经过 $5 \% \mathrm{HF}$ 处理 $3 \mathrm{~min}$ 的 SiNWs 超声分散在 $2 \mathrm{~mL}$ 乙醇中, 并将其浸入到 $\mathrm{SDS} /$ 金 属离子水溶液中，反应 $5 \mathrm{~min}$ ，乙醇清洗.

\section{$4.2 \mathrm{AuNPs} / \mathrm{SiNWs}$ 作催化剂催化还原亚甲基蓝}

将 $0.1 \mathrm{~mL}$ 的 $2.5 \times 10^{-3} \mathrm{~mol} / \mathrm{L}$ 亚甲基蓝与 $2.8 \mathrm{~mL}$, $\mathrm{pH}=9.2$ 的 $\mathrm{Na}_{2} \mathrm{CO}_{3} / \mathrm{NaHCO}_{3}$ 缓冲溶液混合, 用 $\mathrm{N}_{2}$ 吹扫 5 $\min$ 后, 测吸收光谱 $\left(A_{0}\right)$. 随后加入 $5 \mathrm{mg}$ 刚制得的 AuNPs/SiNWs 复合材料的催化剂以及 $0.15 \mathrm{~mL} 0.01$ $\mathrm{mol} / \mathrm{L} \mathrm{NaBH} 4$, 在反应过程中测吸收光谱 $\left(A_{\mathrm{t}}\right)^{[16]}$.

\section{References}

[1] Chen, X.; He, J.; Yan, C.; Tang, H. J. Phys. Chem. B 2006, 110 21684.

[2] Dong, S.-A.; Liu, F.; Hou, S.-Q.; Pan, Z.-F. Acta Chim. Sinica 2010 68(15), 1519. (董守安, 刘锋, 侯树谦, 潘再富, 化学学报, 2010, 68(15), 1519.)

[3] Zhang, Q. H.; Gao, L.; Zheng, S. Acta Chim. Sinica 2001, 59, 1908. (张青红, 高濂, 郑珊, 化学学报, 2001, 59, 1908.)

[4] Zhang, Q.; Yao, Z.-Q.; Zhou, R.; Du, Y.-K.; Yang, P. Acta Chim. Sinica 2012, 70, 2149. (张强, 姚章权, 周蓉, 杜玉扣, 杨平, 化学 学报, 2012, 70, 2149.)

[5] Kim, J.; Roberts, G. W.; Kiserow, D. J. Chem. Mater. 2006, 18, 4710.

[6] Yi, D. K.; Lee, S. S.; Ying, J. Y. Chem. Mater. 2006, 18, 2459.

[7] Pol, V. G.; Grisaru, H.; Gedanken, A. Langmuir 2005, 21, 3635.

[8] Jiang, Y.; Gao, Q. M. J. Am. Chem. Soc. 2006, 128, 716.

[9] Mitsudome, T.; Nose, K.; Mori, K.; Mizugaki, T.; Ebitani, K.; Jitsukawa, K.; Kaneda, K. Angew. Chem., Int. Ed. 2007, 46, 1.

[10] Yasseri, A. A.; Sharna, S.; Kamins, T. I.; Li, Z.; Williama, R. S. Appl. Phys. A 2006, 82, 659 .

[11] Fang, C.; Agarwal, A.; Widjaja, E.; Garland, M. V.; Wong, S. M.; Linn, L.; Khalid, N. M. Chem. Mater. 2009, 21, 3542. 
[12] Sun, X. H.; Sammynaiken, R.; Naftel, S. J.; Tang, Y. H.; Zhang, P.; Kim, P.-S.; Sham, T. K.; Fan, X. H.; Zhang,Y.-F.; Lee, C. S.; Lee, S. T.; Wong, N. B.; Hu, Y.-F.; Tan, K. H. Chem. Mater. 2002, 14, 2519.

[13] Sun, X. H.; Li, C. P.; Wong, N. B.; Lee, C. S.; Lee, S. T. Inorg. Chem. 2002, 41, 4331.

[14] Peng, K. Q.; Wang, X. X.; Wu, L.; Lee, S. T. Nano Lett. 2009, 9, 3704.

[15] Sun, X. H.; Peng, H. Y.; Tang, Y. H.; Shi, W. S.; Wong, N. B.; Lee, C. S.; Lee, S. T.; Sham, T. K. J. Appl. Phys. 2000, 89, 6396.

[16] Hu, H.; Shao, M.; Zhang W.; Lu, L.; Wang, H.; Wang, S. J. Phys. Chem. C 2007, 111(8), 3467.

[17] Zhang, P.; Zhou, X. T.; Tang, Y. H.; Sham, T. K. Langmuir 2005, 21,8502 .

[18] Yin, H. F.; Ma, Z.; Chi, M. F.; Dai, S. Catal. Lett. 2010, 136(3 4), 209.

[19] Deng, J. P.; Shih, W. C.; Mou, C. Y. J. Phys. Chem. C 2007, 111, 9723.

[20] Deng, J. P.; Shih, W. C.; Mou, C. Y. ChemPhysChem 2005, 6, 2021.

[21] Jana, N. R.; Pal, T. Langmuir 1999, 15, 3458.

[22] Pradhan, N.; Pal, A.; Pal, T. Langmuir 2001, 17, 1800.
[23] Hayakawa, K.; Yoshimura, T.; Esumi, K. Langmuir 2003, 19, 5517.

[24] Esumi, K.; Isono, R.; Yoshimura, T. Langmuir 2004, 20, 237.

[25] Guo, Y.-L.; Yan, H.-T.; Pei, R.-H. J. Northwest Univ. 2007, 5(1), 1. (郭艳丽，阎宏涛，裴若会，西北大学学报, 2007, 5(1), 1.)

[26] Tao, A.; Kim, F.; Hess, C.; Goldberger, J.; He, R.; Sun, Y.; Xia, Y.; Ynag, P. Nano Lett. 2003, 3, 1229.

[27] Qi, J. F.; Masumoto, Y. Mater. Res. Bull. 2001, 36, 1407.

[28] Sun, X. P.; Wei, W. T. Langmuir 2010, $26(9), 6133$.

[29] Zhang, X.-D.; Cao, Y.; He, J.-H. Acta Chim. Sinica 2009, 67, 1277. (张晓丹, 曹阳, 贺军辉, 化学学报, 2009, 67, 1277.)

[30] He, J. H.; Ichinose, I.; Kunitake, T.; Nakao, A. Langmuir 2002, 18, 10005.

[31] He, J. H.; Kunitake, T.; Nakao, A. Chem. Mater. 2003, 15, 4401.

[32] Shi, W. S.; Peng, H. Y.; Zheng, Y. F.; Wang, N.; Shang, N. G.; Pan, Z. W.; Lee, C. S.; Lee, S. T. Adv. Mater. 2000, 12, 1343.

[33] Shao, M. W.; Wang, H.; Zhang, M. L.; Ma, D. D. D.; Lee, S. T. Appl. Phys. Lett. 2008, 93, 243110.

[34] Lee, S. T.; Wang, N.; Zhang, Y. F.; Tang, Y. H. Mater. Res. Bull. 1999, 24, 36.

[35] Zhang, Y. F.; Tang, Y. H.; Lam, C.; Wang, N.; Lee, C. S.; Bello, I.; Lee, S. T. J. Cryst. Growth 2000, 212, 115.

(Qin, X.) 